

\title{
Determinación del Modelo de Saturación de Agua Inicial basado en Curvas de Presión Capilar por Tipo de Roca
}

\author{
Eddymar Márquez ${ }^{1}$ (D) César Aguilar ${ }^{1}$, Américo Perozo² \\ ${ }^{1}$ Estudios Integrados de Yacimientos Occidente. Petróleos de Venezuela (PDVSA). Maracaibo, \\ Venezuela. \\ ${ }^{2}$ Facultad de Ingeniería, División de Estudios para Graduados, CP. 4001. Universidad del Zulia. \\ Maracaibo, Venezuela. \\ *Autor de correspondencia:marquezeddymar@ gmail.com; aguilarcp9@ gmail.com \\ https://doi.org/10.22209/rt.v44n3a01 \\ Recepción: 04 de noviembre de 2020 | Aceptación: 04 de junio de 2021 | Publicación: 01 de agosto 2021
}

\section{Resumen}

La saturación de agua inicial $\left(\mathrm{S}_{\mathrm{wi}}\right)$ a partir de registros está influenciada por el drenaje de fluidos de los pozos productores, generando subestimación del petróleo original en sitio (POES). Para restaurar las condiciones iniciales del yacimiento, es necesario utilizar pruebas de presión capilar $\left(\mathrm{P}_{\mathrm{c}}\right)$ de drenaje, que determinan la distribución de $S_{\text {wi }}$ previa a cualquier producción de hidrocarburos. Esta investigación tuvo como objetivo determinar el modelo de $S_{w i}$, basado en curvas de $P_{c}$ por tipo de roca, para una mejor estimación del POES del yacimiento LUZ de la cuenca de Maracaibo. El procedimiento metodológico incluyó: recopilación de datos (registros y núcleos, con 15 muestras de $\mathrm{P}_{\mathrm{c}}$ ), descripción de tipos de roca, determinación del modelo de $\mathrm{S}_{\mathrm{wi}}$, y estimación del POES. Entre los resultados, destacan: el modelo J-Leverett se ajustó mejor a las curvas de $\mathrm{P}_{\mathrm{c}}$ del yacimiento para todos los tipos de roca; el POES estimado utilizando la saturación de agua $\left(S_{\mathrm{w}}\right)$ del modelo propuesto basado en presión capilar y la calculada usando registros, mostró un 19,8 \% de discrepancia, evidenciando la importancia de un modelo robusto para incrementar la certidumbre en el cálculo de reservas.

Palabras clave: modelo; petróleo original en sitio; presión capilar; saturación de agua inicial; tipo de roca.

\section{Determination of the Initial Water Saturation Model based on Capillary Pressure Curves by Rock Type}

\begin{abstract}
The log-derived initial water saturation $\left(S_{\mathrm{wi}}\right)$ is influenced by fluids drainage from the producing wells, generating underestimation of the Stock-Tank Original Oil in Place (STOOIP). To restore the initial conditions of the reservoir, it is necessary to use drainage Capillary Pressure $\left(\mathrm{P}_{\mathrm{c}}\right)$ tests, which determine the distribution of $\mathrm{S}_{\mathrm{wi}}$, prior to any hydrocarbon production. This research aimed to determine the $S_{w i}$ model, based on $P_{c}$ curves by rock type, for a better estimation of the STOOIP of LUZ reservoir in the Maracaibo Basin. The methodological procedure included: data gathering (logs and cores, with 15 plug samples for $\mathrm{P}_{c}$ analysis), description of rock types, determination of the $\mathrm{S}_{\mathrm{wi}}$ model and estimation of the STOOIP. Among the results, the following stand out: the J-Leverett model fit best to the $P_{c}$ curves of the reservoir for all rock types; the estimated STOOIP using the water saturation $\left(S_{w}\right)$ of the proposed capillary pressure based model and the one estimated using logs, showed a discrepancy of $19.8 \%$, evidencing the importance of a robust model to increase certainty in the estimation of reserves.
\end{abstract}

Keywords: capillary pressure; initial water saturation; model; rock type; stock-tank original oil in place. 


\section{Introducción}

Para la estimación del POES, se requiere conocer la $S_{w}$ a las condiciones iniciales del yacimiento. Registros de pozos (resistividad) suelen estar afectados por el drenaje de fluidos del yacimiento; adicionalmente, las curvas de resistividad antiguas tenían problemas de no estar enfocadas y tener una pobre resolución vertical (Rider y Kennedy, 2011), por lo que resultan convenientes los experimentos de laboratorio para representar la historia de saturación del yacimiento o el fenómeno de histéresis, siendo los análisis especiales de núcleos, como las pruebas de $\mathrm{P}_{\mathrm{c}}$ de drenaje, capaces de simular las condiciones iniciales del yacimiento (Valenti et al., 2002).

Según Valenti et al. (2002), cuando se observan en conjunto las curvas de $\mathrm{P}_{\mathrm{c}}$, se aprecian distintas formas de las mismas, así como dispersión de datos, representando la heterogeneidad del yacimiento. Este comportamiento sugiere que los datos deben ser clasificados de acuerdo a la calidad de roca de la muestra (Obeida et al., 2005; Xu y Torres, 2012).

El propósito de esta investigación fue la determinación del modelo de $S_{w i}$, basado en $P_{c}$ por tipo de roca, de un yacimiento siliciclástico en la cuenca de Maracaibo, para mejorar el cálculo del POES. Los resultados se basaron en el procesamiento y análisis de datos de núcleos y registros; estos consistieron en la descripción de los tipos de roca presentes en el yacimiento, clasificación de las curvas de $\mathrm{P}_{\mathrm{c}}$ por tipo de roca, selección del modelo que mejor se ajustó y representó los datos del yacimiento, generación de ecuaciones de saturación de agua, comparación de las curvas de $S_{\mathrm{w}}$ del modelo propuesto con la derivada de registros en los primeros pozos perforados, así como también contraste del POES en un área del yacimiento, obtenido a partir de la $S_{\mathrm{w}}$ del modelo, con el calculado a partir de la $\mathrm{S}_{\mathrm{w}}$ derivada de registros (Obeida et al., 2005; Paradigm y Epos, 2011; Xu y Torres, 2012).

\section{Materiales y Métodos}

\section{Fase I: recopilación y validación de la información}

Se recopilaron y validaron datos del yacimiento (por normas de confidencialidad de la empresa PDVSA, los nombres originales del yacimiento, área de estudio y pozos han sido cambiados), de los pozos con núcleo, entre los que destacan análisis rutinarios o convencionales de núcleos (RCA), para determinar tipos de roca y análisis especiales de núcleo (SCAL), como las pruebas de $\mathrm{P}_{\mathrm{c}}$ de drenaje para la determinación del modelo de $\mathrm{S}_{\mathrm{wi}}$, así como registros convencionales de pozos. Se generó una base de datos robusta utilizando un programa de petrofísica.

\section{Fase II: descripción de los tipos de roca con base en parámetros estadísticos}

Se utilizó la metodología del indicador de zonas de flujo (FZI) de Amaefule et al. (1993), con base en datos de porosidad $(\phi)$ y permeabilidad $(\mathrm{k})$, corregidos por presión de sobrecarga, de acuerdo con Jones (1988). El FZI fue calculado para todas las muestras mediante las Ecuaciones 1, 2 y 3 (Amaefule et al., 1993), y los resultados fueron analizados utilizando herramientas estadísticas, las cuales permitieron identificar los tipos de roca presentes en el yacimiento.

$$
\text { Índice de calidad de yacimiento: RQI }(\mu \mathrm{m})=0,0314 * \sqrt{\frac{\mathrm{k}}{\phi_{\mathrm{e}}}}
$$

Donde, $\phi_{\mathrm{e}}$ : porosidad efectiva (fracción); k: permeabilidad (md)

$$
\text { Índice de porosidad normalizada: } \phi_{\mathrm{z}}(\text { fracción })=\frac{\phi_{\mathrm{e}}}{\left(1-\phi_{\mathrm{e}}\right)}
$$

Indicador de zona de flujo: FZI $(\mu \mathrm{m})=\frac{\mathrm{RQI}}{\phi_{\mathrm{z}}}$

\section{Fase III: preparación de los datos de $P_{\mathrm{c}}$ y su relación con las propiedades petrofísicas de núcleo}

En esta fase se clasificaron los datos obtenidos de las pruebas de $\mathrm{P}_{\mathrm{c}}$ de drenaje por tipo de roca; previamente, se realizaron correcciones de los datos obtenidos de las pruebas de $\mathrm{P}_{\mathrm{c}}$ de laboratorio y las mismas se convirtieron a condiciones de yacimiento. 
Se detallan a continuación las ecuaciones para hacer las correcciones por presión de sobrecarga señaladas por Paradigm y Epos (2011):

$$
\mathrm{P}_{\mathrm{c}} \text { corregida por presión de sobrecarga: } \mathrm{P}_{\mathrm{c}_{\mathrm{corr}}}(\mathrm{lpc})=\frac{\mathrm{P}_{\mathrm{c}_{\mathrm{lab}}}}{\sqrt{\frac{\phi_{\mathrm{yac}}}{\phi_{\text {lab }}}}}
$$

Donde, $\mathrm{P}_{\mathrm{c}_{\mathrm{lab}}}$ : presión capilar a condiciones de laboratorio (lpc); $\Phi_{\mathrm{yac}}$ : porosidad a condiciones iniciales del yacimiento (fracción); $\phi_{\mathrm{lab}}$ : porosidad a condiciones de laboratorio (fracción).

$$
\mathrm{S}_{\mathrm{w}} \text { corregida por presión de sobrecarga: } \mathrm{S}_{\mathrm{w}_{\mathrm{corr}}}(\text { fracción })=1-\left(1-\mathrm{S}_{\mathrm{w}_{\mathrm{lab}}}\right) * \frac{\phi_{\mathrm{yac}}}{\phi_{\mathrm{lab}}}
$$

Donde, $\mathrm{S}_{\mathrm{w}_{\text {lab }}}$ : saturación de agua a condiciones de laboratorio (fracción).

Ecuaciones para la conversión de los datos del sistema utilizado en el laboratorio al sistema yacimiento (Paradigm y Epos, 2011):

Presión capilar convertida al sistema yacimiento: $\mathrm{P}_{\mathrm{c}_{\mathrm{yac}}}(\mathrm{lpc})=\mathrm{P}_{\mathrm{c}_{\operatorname{corr}}} \frac{(\sigma * \cos \theta)_{\mathrm{yac}}}{(\sigma * \cos \theta)_{\mathrm{lab}}}$

Donde, $(\sigma * \cos \theta)_{\text {yac }}=$ tensión interfacial $*$ coseno del ángulo de contacto a condiciones iniciales del yacimiento igual a $26 \mathrm{din} / \mathrm{cm}$ para el sistema presente (agua-petróleo), según Adams y Van den Oord, (1993); $(\sigma * \cos \theta)_{\mathrm{lab}}=$ tensión interfacial * coseno del ángulo de contacto a condiciones de laboratorio.

Fase IV: determinación del modelo de $S_{\mathrm{wi}}$ del yacimiento $\mathrm{LUZ}$ a partir de las pruebas de $\mathrm{P}_{\mathrm{c}}$ por tipo de roca

A continuación se detallan los pasos seguidos:

a) Cálculo de la curva de $P_{c}$ versus $S_{w i}$, por tipo de roca, para cada uno de los modelos más usados dentro de la literatura (Adams y Van den Oord, 1993; Paradigm y Epos, 2011) en los pozos con núcleo.

b) Selección del modelo que mejor se ajustó a las curvas de $\mathrm{P}_{\mathrm{c}}$ de núcleo para cada tipo de roca, ajustando los coeficientes propuestos por los autores originales (Paradigm y Epos, 2011).

c) Predicción de la curva de $S_{\text {wi }}$ por encima del nivel de agua libre (FWL). Para ello, se determinó la altura por encima del FWL $(\mathrm{H})$ para cada punto. Una vez obtenida la altura, se calculó la $\mathrm{P}_{\mathrm{c}}$ a cada profundidad, de acuerdo a Obeida et al., (2005):

$\mathrm{H}($ pies $)=\mathrm{FWL}-\mathrm{TVD}_{\mathrm{ss}}$

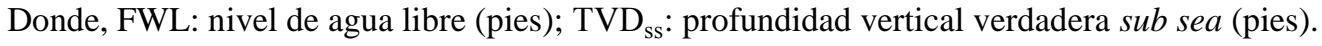

$\mathrm{P}_{\mathrm{c}}(\mathrm{lpc})=0,433 * \mathrm{H} *\left(\rho_{\text {agua }}-\rho_{\text {petróleo }}\right)$

Donde, $\rho_{\text {agua }}:$ densidad del agua $\left(\mathrm{g} / \mathrm{cm}^{3}\right) ; \rho_{\text {petróleo }}$ : densidad del petróleo $\left(\mathrm{g} / \mathrm{cm}^{3}\right)$.

d) Comparación de la curva de $S_{w i}$ obtenida de pruebas de $P_{c}$ y la calculada con la información de registros de los primeros pozos perforados en el área de estudio (PDVSA, 2019).

e) Propagar el modelo a todos los pozos del área de estudio.

Fase V: estimación del POES del área P-1, en la arena basal del yacimiento

Se calculó el POES mediante el método volumétrico (PDVSA, 2005), utilizando la $S_{\mathrm{w}}$ basada en el modelo propuesto de $\mathrm{P}_{\mathrm{c}}$, así como la $\mathrm{S}_{\mathrm{w}}$ derivada de registros, estableciendo el nivel de discrepancia entre ellas.

\section{Fase I: recopilación, validación y procesamiento de la información}

\section{Resultados y Discusión}

Los datos obtenidos de análisis convencionales y especiales de núcleos de 3 pozos se muestran en la Tabla 1, a partir de los cuales se puede ver el número total de muestras de análisis convencionales que sirvieron para la 
determinación de los tipos de roca, así como también de los análisis especiales de las pruebas de $\mathrm{P}_{\mathrm{c}}$ utilizadas para modelar la altura de la $S_{w}$, especificando el tipo de prueba y los sistemas de fluidos manejados en el laboratorio.

Tabla 1. Inventario de las pruebas de $\mathrm{P}_{\mathrm{c}}$ para el yacimiento estudiado.

\begin{tabular}{c|c|ccc}
\hline \multicolumn{1}{c}{ Pozo } & $\begin{array}{c}\text { Análisis } \\
\text { convencionales de } \\
\text { núcleo }(\boldsymbol{\phi} \mathbf{~ y ~ k )}\end{array}$ & \multicolumn{2}{c}{$\begin{array}{c}\text { Análisis especiales de núcleo } \\
\text { Presiones capilares (drenaje) }\end{array}$} \\
\hline \hline & $\mathbf{N}^{\circ}$ de muestras & $\mathbf{N}^{\circ}$ de muestras & Método & $\begin{array}{c}\text { Sistema de fluido } \\
\text { utilizado en el } \\
\text { laboratorio }\end{array}$ \\
\hline \hline LUZ1246 & $11^{1}$ & $4^{1}$ & Plato poroso & Aire/salmuera \\
LUZ1348 & $15^{2}$ & $6^{3}$ & Centrífuga & Petróleo/salmuera \\
LUZ1542 & $15^{4}$ & $5^{5}$ & Centrífuga & Petróleo/salmuera \\
Total de muestras & 41 & 15 & & \\
\hline${ }^{1}$ Omni Laboratories de Venezuela (1997); ${ }^{2}$ Core Laboratories Venezuela (2000); ${ }^{3}$ PDVSA (2019); ${ }^{4}$ Omni \\
Laboratories de Venezuela (2007); ${ }^{5}$ Core Laboratories Venezuela (2008).
\end{tabular}

Fase II: descripción de los tipos de roca con base en parámetros estadísticos

Para mostrar los tipos de roca existentes en el yacimiento, se realizó un gráfico log-log RQI vs $\phi_{z}$ (Figura 1), donde se muestran 6 líneas de pendiente unitaria, correspondientes a los 6 tipos de roca del yacimiento; la intersección de estas rectas con la $\phi_{\mathrm{Z}}=1$ suministra un valor aproximado del FZI de cada tipo de roca, ordenados de mayor (mayor FZI) a menor calidad (menor FZI).

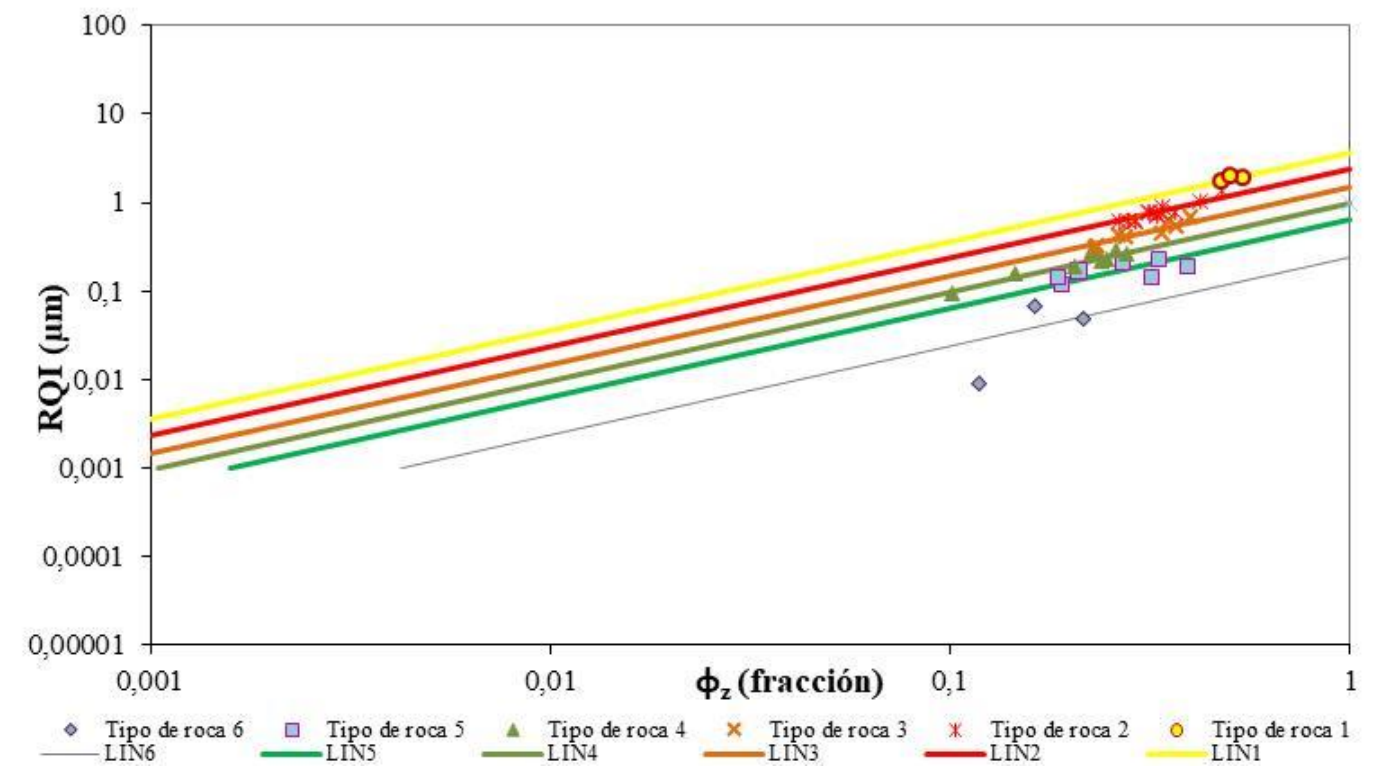

Figura 1. Visualización de los tipos de roca del yacimiento LUZ, mediante el índice de calidad de yacimiento versus el índice de porosidad normalizada.

Algunos indicadores estadísticos del FZI por cada tipo de roca se presentan en la Tabla 2. Se observa que la desviación estándar para cada tipo de roca varía entre "medianamente baja" y "baja", concluyendo por tanto que las clases identificadas son consistentes desde un punto de vista estadístico. Para la propagación de los tipos de roca, se calculó el FZI utilizando la permeabilidad generada mediante el modelo de Timur (Uguru, 2004), con modificaciones en sus coeficientes. 
Tabla 2. Parámetros estadísticos del indicador de zona de flujo, utilizados para la clasificación de los tipos de roca del yacimiento LUZ.

\begin{tabular}{ccccc}
\hline Tipo de roca & FZI-medio & FZI-Mín & FZI-Máx & Desviación estándar \\
\hline 1 & 3,593 & 3,553 & 3,633 & 0,056 \\
2 & 2,347 & 2,101 & 2,844 & 0,217 \\
3 & 1,460 & 1,241 & 1,745 & 0,180 \\
4 & 0,960 & 0,808 & 1,108 & 0,102 \\
5 & 0,630 & 0,447 & 0,781 & 0,141 \\
6 & 0,237 & 0,078 & 0,409 & 0,166 \\
\hline
\end{tabular}

FZI-medio: valor medio del indicador de zonas de flujo; FZI-Mín: valor mínimo del indicador de zonas de flujo; FZI-Máx: valor máximo del indicador de zonas de flujo.

\section{Fase III: preparación de los datos de $P_{\mathrm{c}}$ y su relación con las propiedades petrofísicas de núcleo}

Una vez corregidos los datos de $\mathrm{P}_{\mathrm{c}}$ y convertidos a condiciones de yacimiento, se graficó la saturación de agua irreducible $\left(S_{\text {wirr }}\right.$ ) versus RQI (Figura 2), donde se aprecia que rocas con bajo RQI muestran valores altos de $S_{\text {wirr. }}$ De acuerdo a esto, las variables introducidas por Amaefule et al., (1993), se relacionan con las propiedades físicas del yacimiento, lo cual reafirma cómo físicamente estas controlan la capacidad de flujo y almacenamiento de la roca.

Por otro lado, las curvas de $\mathrm{P}_{\mathrm{c}}$ se clasificaron por tipo de roca, usando el parámetro de FZI (Amaefule et al., 1993). Los tipos de roca 4, 5 y 6 fueron clasificados en el tipo de roca 4, ya que solo se disponía de una muestra de los tipos 5 y 6 , siendo así imposible de modelar los mismos.

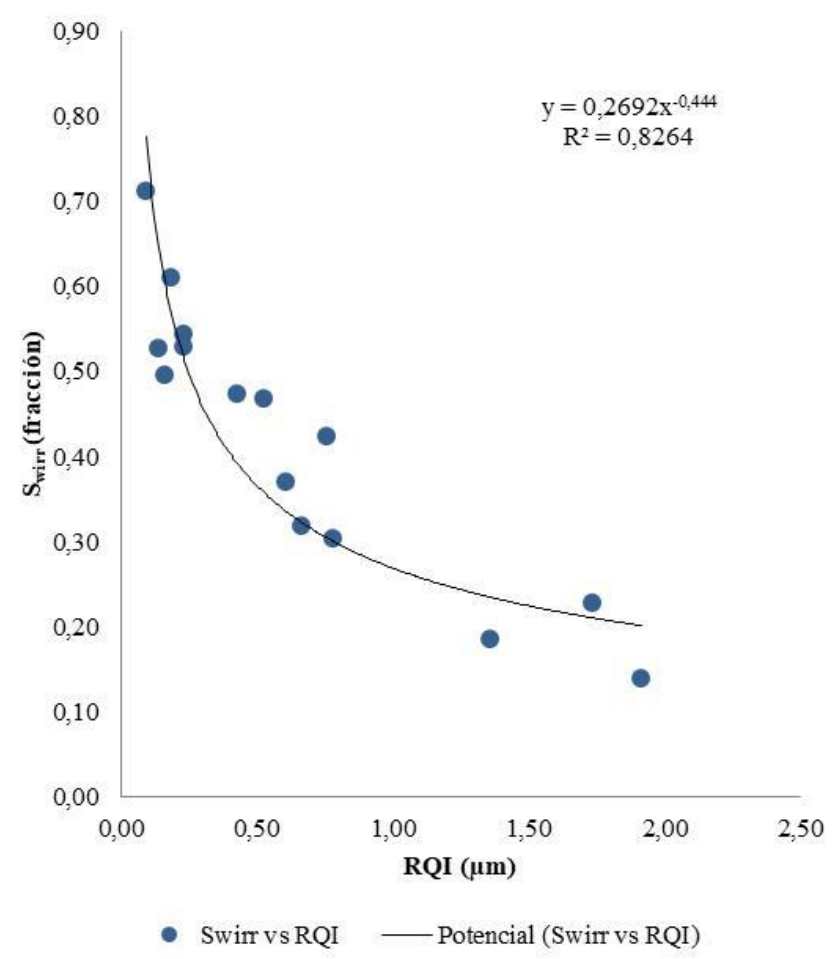

Figura 2. Saturación de agua irreducible de cada muestra de presión capilar de drenaje versus el índice de calidad de yacimiento calculado para cada muestra. 


\section{Fase IV: determinación del modelo de $S_{w i}$ del yacimiento LUZ a partir de las pruebas de $P_{c}$ por tipo de roca}

A manera referencial, se muestra la Figura 3 de la selección del modelo en el tipo de roca 1. A la izquierda de la gráfica se listan las ecuaciones definidas y el error encontrado entre la saturación de agua de cada punto y la modelada por la función fijada. En general, los modelos evaluados generaron errores muy bajos; sin embargo, el modelo de Leverett fue seleccionado porque representa la mejor forma de las curvas y reproduce mejor el valor de $\mathrm{S}_{\text {wirr }}$

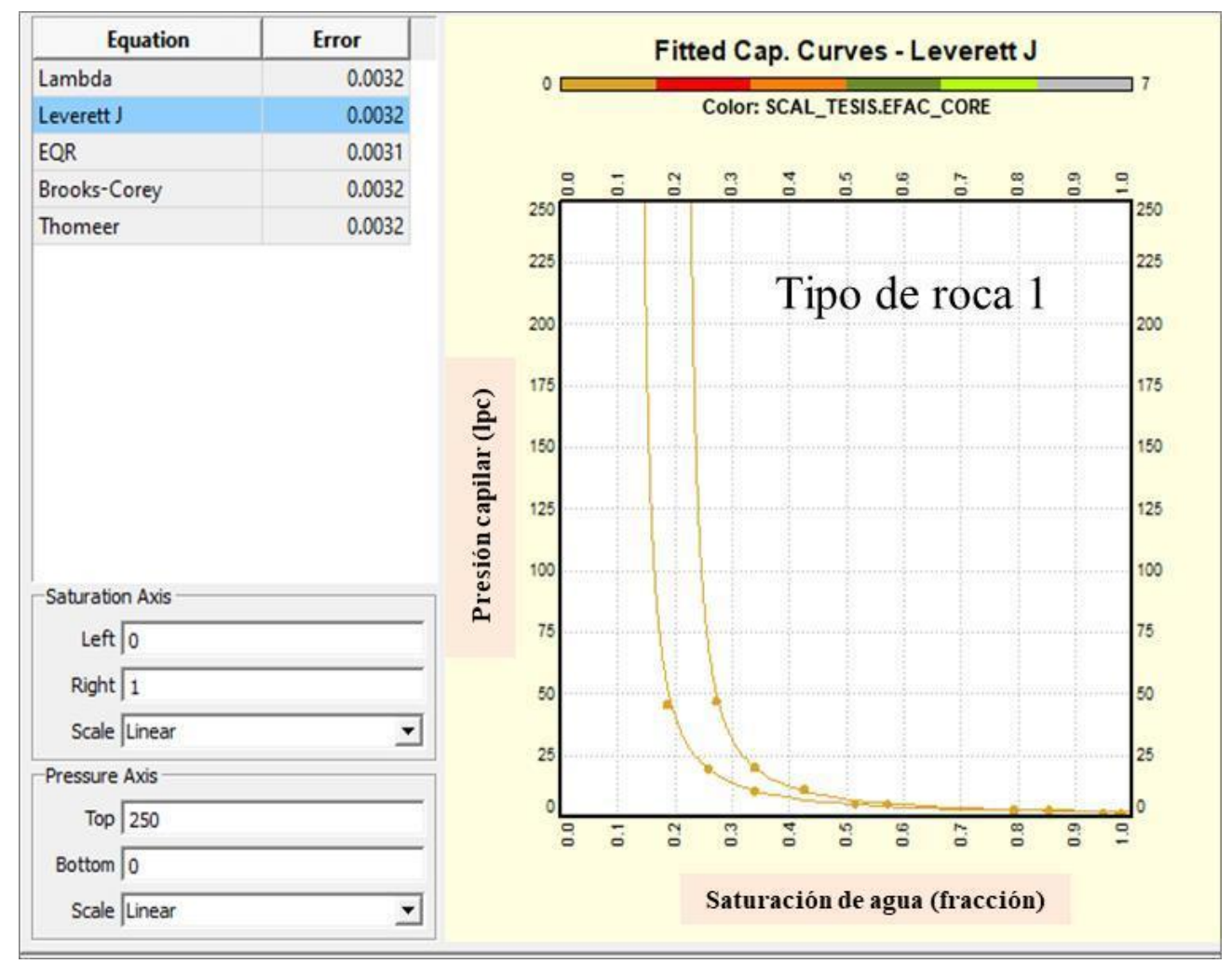

Figura 3. Curvas de $\mathrm{P}_{\mathrm{c}}$ correspondientes al tipo de roca 1 del yacimiento LUZ. Correlación J-Leverett.

Las curvas de $\mathrm{P}_{c}$, modeladas utilizando la función J-Leverett con coeficientes constantes para los diferentes tipos de roca, se muestran en la Figura 4. Los parámetros de ajuste de la ecuación de $S_{w}$ por tipo de roca se obtuvieron con el RQI de cada muestra de $\mathrm{P}_{\mathrm{c}}$, con la ayuda del módulo para ajuste de coeficientes del programa utilizado. Las ecuaciones propuestas se incluyen en la Tabla 3. En la Figura 5, se presenta un gráfico uno a uno entre la $S_{w}$ modelada con Leverett versus la $S_{w}$ de la curva de $P_{c}$ medida en el laboratorio para cada tipo de roca, donde en cada gráfico se muestra una línea de pendiente unitaria que pasa por el origen; a medida que los puntos se acercan a esa tendencia, el modelo tiene un mejor ajuste.

Se representan en 3D los modelos por tipo de roca (Figura 6), de manera que cada modelo predice la $S_{w}$ como una función de $\mathrm{P}_{\mathrm{c}} \mathrm{y}$ valores $\mathrm{Z}$ de RQI. El modelo tiene un buen ajuste a los puntos de los datos, ya que las curvas de $\mathrm{P}_{\mathrm{c}}$ se ubican sobre o cerca de la superficie. En la Figura 6 se muestra la integración de todos los parámetros involucrados en la ecuación de $S_{\mathrm{w}}$ de Leverett, donde cabe mencionar que a medida que el RQI es mayor, la saturación de agua disminuye, pero esta a su vez es más pequeña a medida que la $\mathrm{P}_{\mathrm{c}}$ aumenta. Por lo tanto, la $\mathrm{S}_{\mathrm{w}}$ en un punto específico del yacimiento dependerá de a qué altura por encima del FWL esté; esto se nota cuando se hace la transformación de $\mathrm{P}_{\mathrm{c}}$ a altura. Por otro lado, cada tipo de roca tiene una zona de transición; esto dependerá de la calidad de la misma, es decir, a medida que el RQI es mayor (poros más grandes), la zona de agua más petróleo es más corta. 


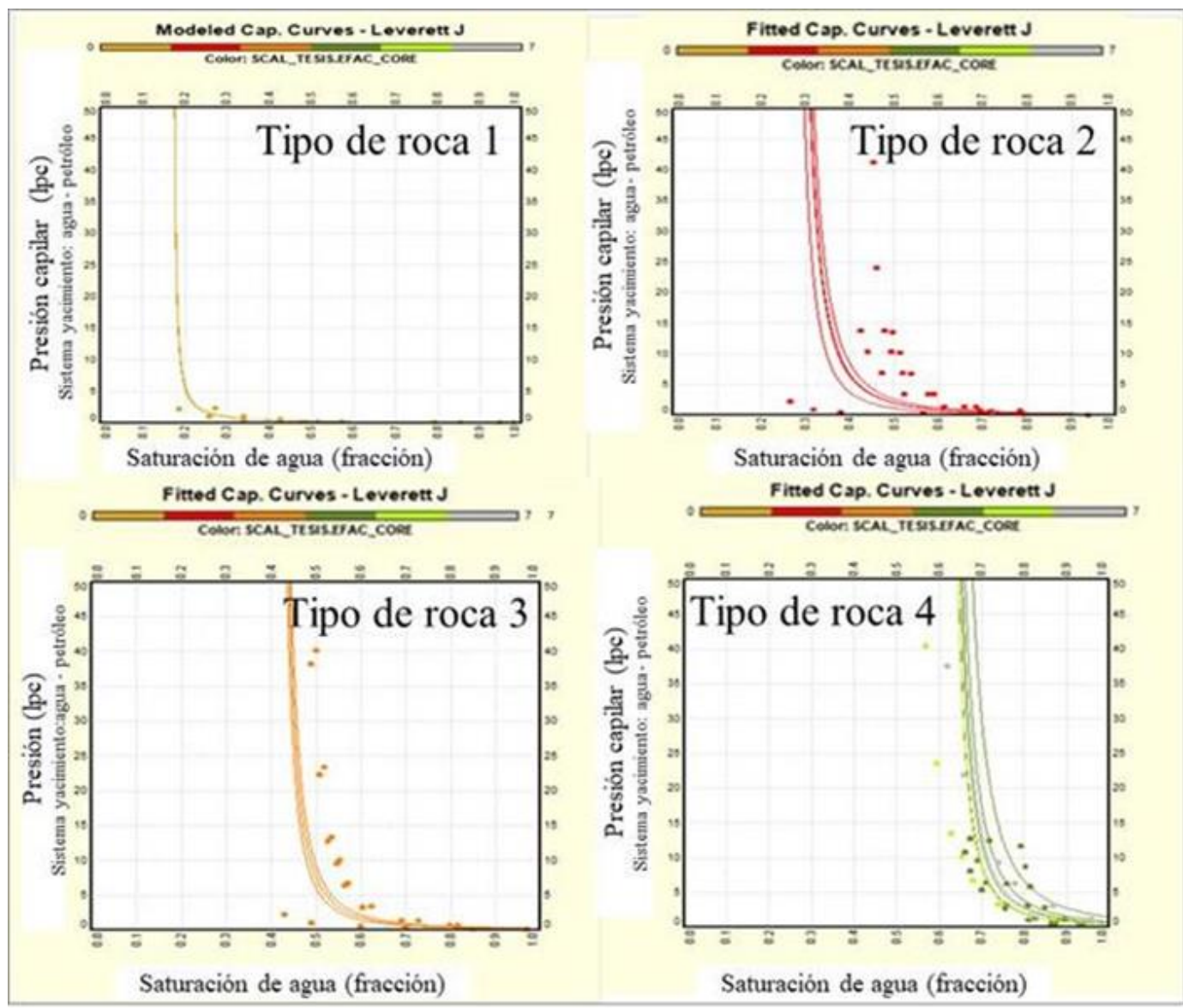

Figura 4. Curvas de Pc del yacimiento LUZ, modeladas con J-Leverett y coeficientes constantes

Tabla 3. Ecuaciones propuestas para determinar la saturación de agua por tipo de roca en el yacimiento LUZ, utilizando el modelo J-Leverett.

Tipo de roca 1

\begin{tabular}{|c|c|c|c|c|}
\hline \multicolumn{5}{|c|}{$\begin{array}{l}\text { Model Sw Equation } \\
\text { Sw Equation } \sqrt{\left.(0.170311)+(1-(0.170311))^{*}(1.165030)^{*}\left(\text { PC } C^{*} \text { SQRT(PERMEABILTY/POROSITY }\right)\right)^{* *}-(0.8}\end{array}$} \\
\hline Parameter & Model & Intercept & Gradient & $\begin{array}{l}\text { Regression } \\
\text { Equation }\end{array}$ \\
\hline SWI & Constant & 0.170311 & & $S W l=0.170311$ \\
\hline A & Constant & 1.165030 & & $A=1.165030$ \\
\hline $\mathrm{N}$ & Constant & 0.809578 & & $\mathrm{~N}=0.809578$ \\
\hline
\end{tabular}

Tipo de roca 3

\begin{tabular}{|c|c|c|c|c|}
\hline \multicolumn{5}{|c|}{ Sw Equation $\sqrt{\left.(0.398200)+(1-(0.398200))^{*}(0.813689) *(P C * S Q R T \text { (PERMEABILITY/POROSITY })\right)^{* *}-(0.475428}$} \\
\hline Parameter & Model & Intercept & Gradient & $\begin{array}{c}\text { Regression } \\
\text { Equation }\end{array}$ \\
\hline SWI & Constant & 0.39820 & & $\mathrm{SWl}=0.398200$ \\
\hline A & Constant & 0.81368 & & $A=0.813689$ \\
\hline $\mathrm{N}$ & Constant & 0.47542 & & $\mathrm{~N}=0.475428$ \\
\hline \multicolumn{4}{|c|}{$\mathrm{S}_{\mathrm{w}}=0,398200+(1-0,398200)^{*} 0,813689 *$} & $\left.* \sqrt{\frac{\mathrm{k}}{\phi}}\right)$ \\
\hline
\end{tabular}

Tipo de roca 2

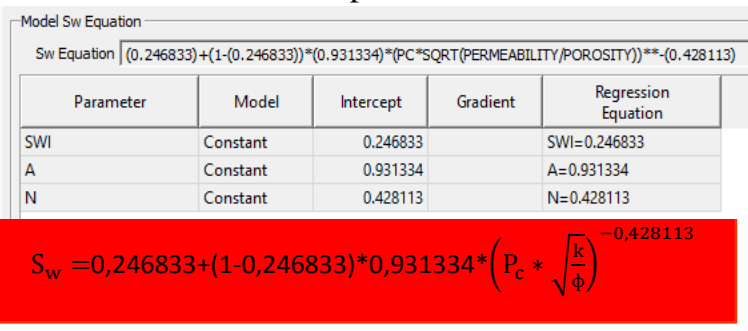

Tipo de roca 4

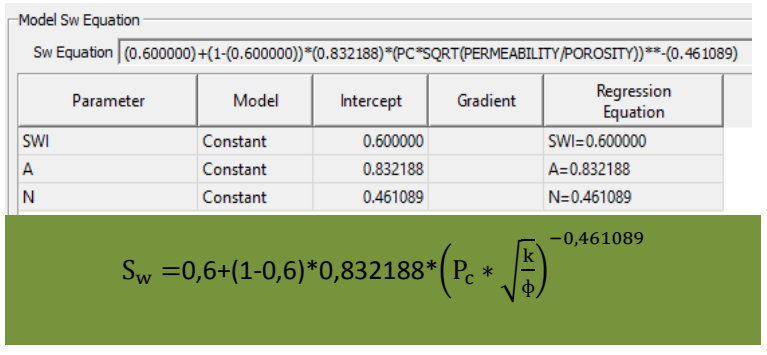




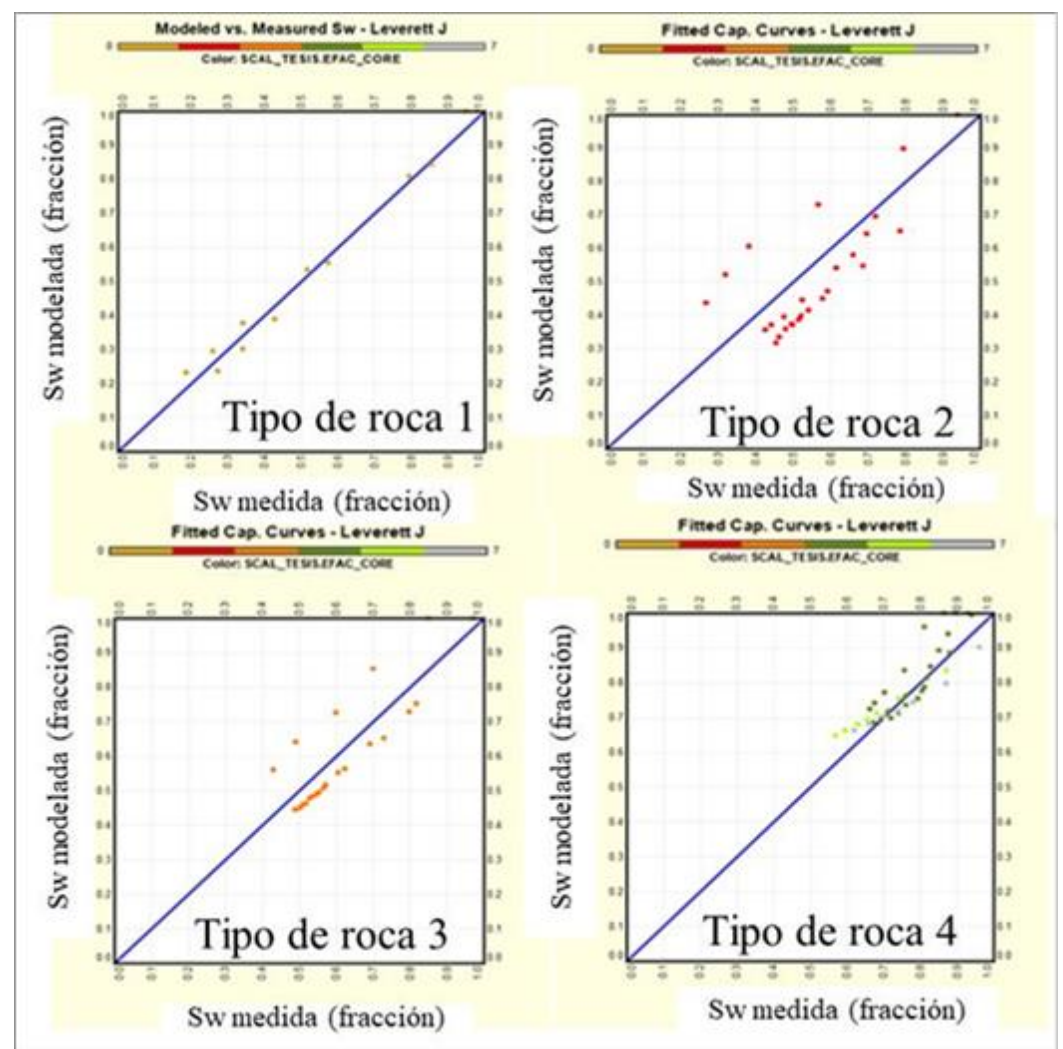

Figura 5. $S_{w}$ modelada de la función J-Leverett versus $S_{w}$ medida en laboratorio, correspondientes al yacimiento LUZ

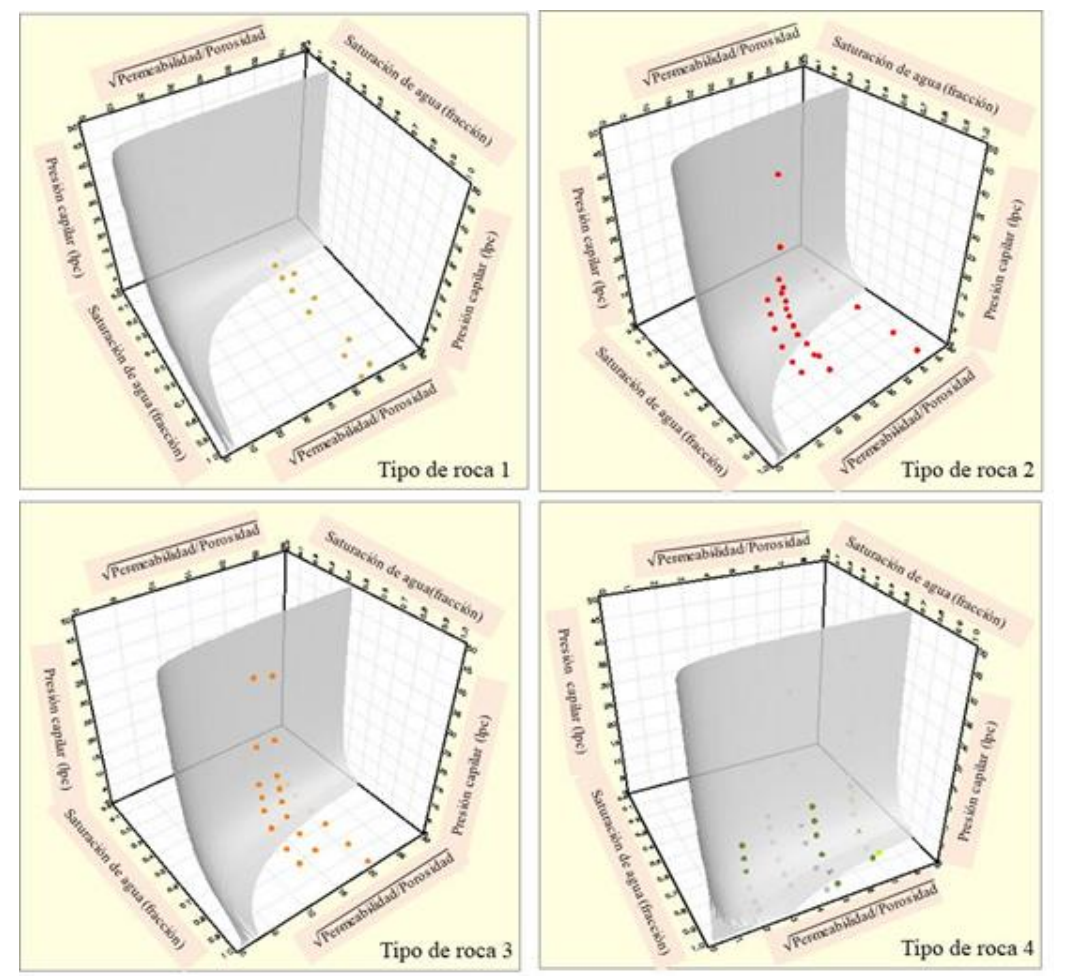

Figura 6. Modelo de $S_{w}$ en 3D para todos los tipos de roca del yacimiento LUZ 
La información de registros de los primeros pozos perforados en el área de estudio (Figura 7) se obtuvo varias décadas antes del año de inicio de la producción del yacimiento LUZ (1992), por lo que dicha información no había sido afectada por el drenaje del yacimiento. Al predecir la $S_{\mathrm{w}}$ por encima del FWL en estos pozos y contrastarla con la información de registros, se puede mencionar lo siguiente: en los pozos LUZ0512 y LUZ0584 hay un buen cotejo entre la curva derivada de $\mathrm{P}_{\mathrm{c}}$ (azul oscuro) y la de registro (azul claro), presentes en la pista 10 de la sábana petrofísica; sin embargo, pequeñas diferencias se presentan en el pozo LUZ0512, ya que se trata de registros de resistividad Long Normal y Short Normal, que por la configuración de electrodos no enfocados, siempre tienen desfase en profundidad. Por otra parte, en el pozo LUZ0267 hay una notable diferencia entre estas dos curvas, debido a la baja resolución vertical de la herramienta de resistividad, viéndose afectada por capas vecinas. Los resultados se consideran satisfactorios y validan el modelo de $S_{w}$ basado en $P_{c} y$, por consiguiente, el rango de amplitud de los tipos de roca.

Como es bien conocido y ha sido referenciado por múltiples autores (Walsh et al., 1993; Whitman, 1995; Griffiths et al., 2000), la calidad de la información de registros de pozos estará en función de factores como resolución vertical de la herramienta y del espesor de la capa; por ejemplo, cuando el espesor de las capas es menor que la resolución vertical de la herramienta, las capas vecinas afectan el valor medido de la propiedad, por lo cual dicho valor no será representativo. Este efecto puede ser visto en los registros viejos (aproximadamente de la década de los 60), especialmente en los registros de Induction de vieja generación y dispositivos no enfocados con resolución vertical muy pobre, que ronda los 8 pies. En los pozos viejos, el tipo de herramienta juega un rol importante, porque la resolución vertical de un registro Dual Laterolog es mejor que la de un registro Induction. Adicionalmente, hay que considerar las propiedades del lodo de perforación; los registros Induction funcionan mejor con lodos a base de agua fresca, mientras que los registros galvánicos, como el Dual Laterolog funcionan mejor cuando el lodo es a base de agua salada. Todo esto indica que no siempre los datos obtenidos de registros son confiables, y la metodología empleada en este trabajo es una opción válida para disminuir la incertidumbre en la cuantificación del POES.

\section{Fase V: estimación del POES del área P-1, en la arena basal del yacimiento}

En la Tabla 4, se muestra la comparación del POES obtenido con la $S_{w}$ basada en $P_{c}$ y el calculado de manera convencional ( $S_{\mathrm{w}}$ derivada de registros). En esta se observa una diferencia de 5,21 MMBN (19,8\%). Esto obedece a que el POES obtenido a partir de la $S_{\mathrm{w}}$ derivada de registros está afectado por el drenaje del yacimiento (esto es observado en pozos nuevos), por lo que no sería el más representativo. Para ilustrar mejor esto, en la Figura 8 , se representan ambos POES y, según estos, se ve disminuido dicho parámetro al este del área, al ver el POES con la $S_{w}$ derivada de registros, mientras que en el POES con $S_{w}$ obtenida con el modelo propuesto, los valores se mantienen altos en esa misma zona. Al utilizar la $S_{\mathrm{w}}$ derivada de registros de todos los pozos asociados, se estaría subestimando el POES, incluso las reservas recuperables de petróleo podrían ser incluso menores que la producción acumulada de petróleo. Para minimizar esos problemas, una mejor cuantificación se obtiene mediante modelos de $\mathrm{P}_{\mathrm{c}}$ dándoles un tratamiento por tipo de roca, tal como lo desarrolla Obeida et al. (2005), así como también Gonzalez et al. (2016). Por lo tanto, una mejor estimación del POES para el área P-1, en la arena basal del yacimiento, resulta en 26,28 MMBN. 

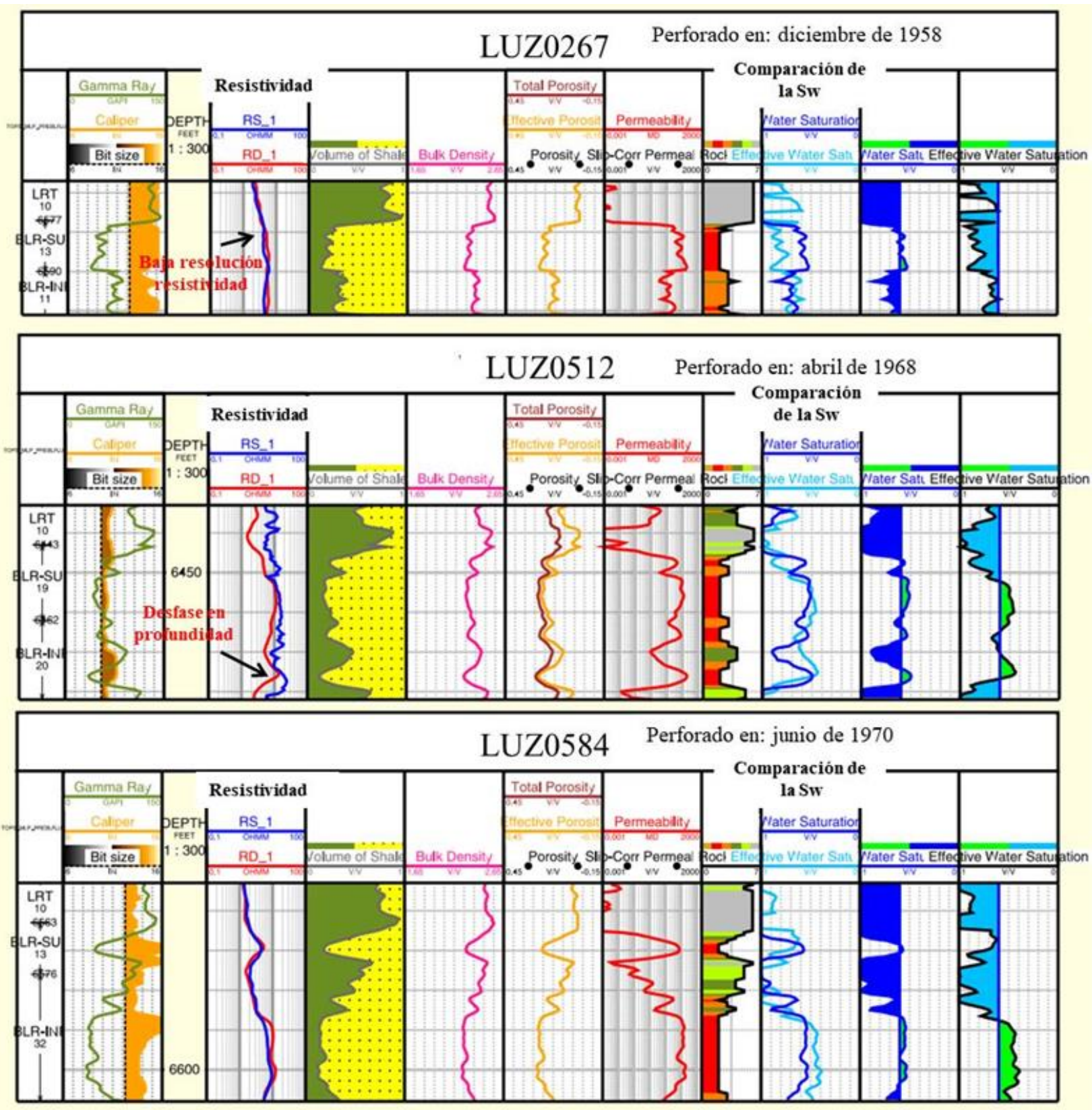

Figura 7. Comparación de la $S_{w}$ obtenida de la curva de $P_{c}$ por encima del FWL y la curva de $S_{w}$ derivada de registros. Primeros pozos perforados del área de estudio 

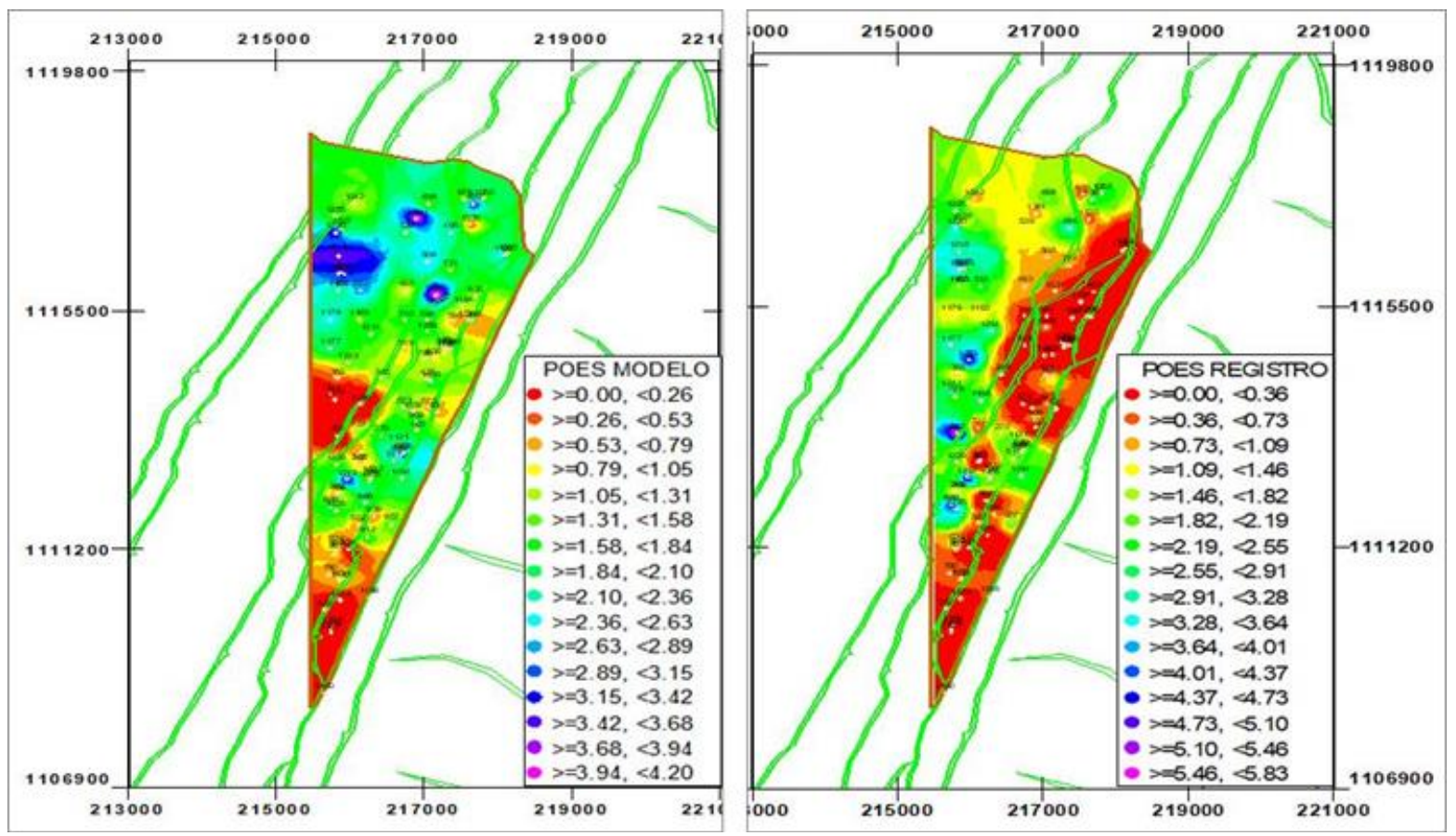

Figura 8. Mapa de POES del área de estudio con $S_{w}$ del modelo propuesto y $S_{w}$ derivada de registros

Tabla 4. Valores medios utilizados en los cálculos de POES del área de estudio.

\begin{tabular}{cccccccc}
\hline $\begin{array}{c}\text { Espesor } \\
\text { medio } \\
\text { (pies) }\end{array}$ & $\begin{array}{c}\text { Porosidad efectiva media } \\
\text { (fracción) }\end{array}$ & $\begin{array}{c}\text { Saturación de agua } \\
\text { media } \\
\text { (fracción) }\end{array}$ & POES \\
Modelo & Registro & Modelo & Registro & Modelo & Registro & Modelo & Registro \\
\hline & & & & & & & \\
10,09 & 8,96 & 0,20 & 0,16 & 0,54 & 0,59 & 26,28 & 21,07 \\
\hline
\end{tabular}

\section{Conclusiones}

En el yacimiento LUZ se identificaron seis tipos de rocas.

El modelo J-Leverett se ajustó mejor a las curvas de $\mathrm{P}_{\mathrm{c}}$ para todos los tipos de roca, por lo que con dicho modelo se establecieron ecuaciones de $S_{\text {wi }}$ para los tipos de roca modelados.

La comparación entre las curvas de $S_{w}$ basada en $P_{c}$ y la derivada de registros en los primeros pozos perforados, mostró un buen ajuste. Las diferencias observadas en algunos de ellos se debieron a problemas asociados a los registros, como efecto de capas vecinas, desfase en profundidad, entre otros.

El POES estimado en el área P-1, en la arena basal del yacimiento, utilizando la $S_{\mathrm{w}}$ del modelo propuesto y el obtenido mediante de la $S_{\mathrm{w}}$ derivada de registro, representa una diferencia de $19,8 \%$, lo cual resalta la importancia de un modelo robusto, tal como el presentado en este trabajo para incrementar la certidumbre en el cálculo de reservas. 


\section{Agradecimiento}

Los autores deseamos expresar nuestro agradecimiento a la empresa PDVSA, Dirección de Exploración y Estudios Integrados de Yacimientos Occidente, por facilitar la información y autorizar la publicación de este trabajo. Igualmente, queremos extender nuestro agradecimiento al Prof. Edgar Pereira (Escuela de Ingeniería de Petróleo de la Universidad del Zulia) por su valiosa colaboración en la realización de este artículo.

\section{Referencias Bibliográficas}

Adams, S., Van den Oord, R. (1993). Petrophysics. Capillary pressure and saturation-height functions. The Hague: Shell International Petroleum Maatschappij B.V.

Amaefule, J., Altunbay, M., Tiab, D., Kersey, D., Keelan, D. (1993). Enhanced reservoir description: using core and log data to identify hydraulic (flow) units and predict permeability in uncored intervals/wells. SPE26436, 68 ${ }^{\text {th }}$ Annual Technical Conference and Exhibition of the Society of Petroleum Engineers (SPE). Texas, USA, 205-220.

Core Laboratories Venezuela, S.A. (2000). Estudio de las propiedades de la roca para PDVSA Exploración y Producción del pozo LUZ1348. Maracaibo: Petróleos de Venezuela (PDVSA).

Core Laboratories Venezuela, S.A. (2008). Estudio de propiedades avanzadas de la roca para PDVSA, pozo LUZ1542. Maracaibo: Petróleos de Venezuela (PDVSA).

González, J., Perozo, A; Medina, F. (2016). Quantification of the Distribution of Initial Water Saturation through Leverett J Function to Calculate Hydrocarbon Reserves. SPE-181177-MS, SPE Latin America and Caribbean Heavy and Extra Heavy Oil Conference. Lima, Peru, 1-14.

Griffiths, R., Barber, T., Faivre, O. (2000). Optimal evaluation of formation resistivities using array induction and array laterolog tools. SPWLA-2000-BBB, SPWLA (Society of Petrophysicists and Well Log Analysts) $41^{\text {st }}$ Annual Logging Symposium. Dallas, USA, 1-13.

Jones, S. (1988). Two-point determinations of permeability and PV vs net confining stress. SPE Formation Evaluation. 3(1), 235-241.

Obeida, T., Al-Mehairi, Y., Suryanarayana, K. (2005).Calculations of fluid saturations from log-derived J-functions in giant complex Middle East carbonate reservoir. IPTC 10057, International Petroleum Technology Conference. Doha, Qatar. 1-5.

Omni Laboratories de Venezuela, C.A. (1997). Reporte final de análisis especiales de núcleos, pozo LUZ1246. Maracaibo: Petróleos de Venezuela (PDVSA).

Omni Laboratories de Venezuela, C.A. (2007). Reporte final de análisis convencionales de núcleos, pozo LUZ1542. Maracaibo: Petróleos de Venezuela (PDVSA).

Paradigm $^{\mathrm{TM}}$; Epos ${ }^{\circledR}$ 4.1 Data Management (2011). Core analysis. Módulo de ayuda del programa Geolog 7. Houston: Emerson.

PDVSA. (2005). Manual del participante en el curso de OFM (oilfield manager) intermedio-avanzado v-2005. Maracaibo: Petróleos de Venezuela (PDVSA).

PDVSA. (2019). Base de datos del proyecto de caracterización petrofísica del yacimiento LUZ. Maracaibo: Dirección de exploración y estudios integrados de yacimientos Occidente, Petróleos de Venezuela (PDVSA).

Rider, M., Kennedy, M. (2011). The geological interpretation of well logs. Third edition. Glasgow: Rider-French Consulting. 
Uguru, C., Udofia, A., Oladiran, O. (2004). Estimating irreducible water saturation and relative permeability from Logs. SPE 140623, 34 ${ }^{\text {th }}$ Annual SPE International Conference and Exhibition. Calabar, Nigeria, 1-6.

Valenti, N., Valenti, R., Koederitz, L. (2002). A unified theory on residual oil saturation and irreducible water saturation. SPE 77545, Annual Technical Conference and Exhibition. Texas, USA, 1-6.

Walsh, J., Brown, S., Asquith, G. (1993). Analyzing old electric logs in shaly sand formations. SPE-25508-MS, SPE Production Operations Symposium. Oklahoma, USA. 919-926.

Whitman, W. (1995) Interpretation of unfocused resistivity logs. Petrophysics - The SPWLA Journal of Formation Evaluation and Reservoir Description, 36(1), 35 -39.

$\mathrm{Xu}, \mathrm{C}$. , Torres, C. (2012). Saturation height and invasion consistent hydraulic rock typing using multi-well conventional logs. 53 ${ }^{\text {rd }}$ SPWLA Annual Logging Symposium. Cartagena, Colombia, 1-16. 


\section{REVISTA TECNICA}

DE LA FACULTAD DE INGENIERIA

UNIVERSIDAD DEL ZULIA

Vol. 44. $\mathbf{N}^{\circ} 3$, Septiembre - Diciembre, 2021

Esta revista fue editada en formato digital y publicada en Agosto 2021, por el Fondo Editorial Serbiluz, Universidad del Zulia. Maracaibo-Venezuela

www.luz.edu.ve

www.serbi.luz.edu.ve

www.produccioncientificaluz.org 